\title{
Survey on Various Gesture Recognition Technologies and Techniques
}

\author{
Noor Adnan Ibraheem \\ Department of Computer Science \\ A.M.U., Aligarh \\ Uttar Pradesh, India
}

\author{
RafiqulZaman Khan \\ Department of Computer Science \\ A.M.U., Aligarh \\ Uttar Pradesh, India
}

\begin{abstract}
Gestures considered as the most natural expressive way for communications between human and computers in virtual system. Hand gesture is a method of non-verbal communication for human beings for its freer expressions much more other than body parts. Hand gesture recognition has greater importance in designing an efficient human computer interaction system. Using gestures as a natural interface benefits as a motivation for analyzing, modeling, simulation, and recognition of gestures. In this paper a survey on various recent gesture recognition approaches is provided with particular emphasis on hand gestures. A review of static hand posture methods are explained with different tools and algorithms applied on gesture recognition system, including connectionist models, hidden Markov model, and fuzzy clustering. Challenges and future research directions are also highlighted.
\end{abstract}

\section{General Terms}

Gesture Recognition System, Hand Gesture Technologies, Hand Gesture Approaches.

\section{Keywords}

Hand Posture, Hand Gesture, Fuzzy Clustering, Artificial Neural Network, Hidden Markov Model, Orientation Histogram.

\section{INTRODUCTION}

Gestures and face expressions easily used for daily humans interactions [1] while human computer interactions still require understanding and analyzing signals to interpret the desired command that made the interaction sophisticated and unnatural [1]. Recently the designing of special input devices witnessed great attention in this field to facilitate the interaction between humans and computers [2], and to accomplish more sophisticated interaction through the computer [2]. It is worth to mention that the window manager is the earlier user interface to communicate with computers [3]. The combining of traditional devices mouse and keyboard with the new designed interaction devices such as gesture and face recognition, haptic sensors, and tracking devices provides flexibility in tele-operating [2], text editing [4], robot control [2], cars system control [2], gesture recognition [4], Virtual Reality (VR) [5], and multi-media interfaces [4], video games [4].Gesture considered as a natural way of communication among people especially hear-impaired [6]. Gestures can be defined as a physical movement [6] of hands, arms, or body that delivers an expressive message [6], and gesture recognition system used to interpret and explain this movement as meaningful command [6][7].

Gesture recognition has been applied in a large range of application areas such as recognizing sign language [6][8], human computer interaction (HCI) [6][9], robot control [6], smart surveillance [6], lie detection [6], visual environments manipulating [6], etc.

Different techniques and tools have been applied for handling gesture recognition, vary between mathematical models like Hidden Markov Model (HMM) [6][10] and Finite State Machine (FSM) [6][11] to approaches based on software computing methods such as fuzzy clustering [12], Genetic Algorithms (GAs) [13] and Artificial Neural Network (ANN) [14]. Hand posture recognition sill an open research area [15], since the human hand is a complex articulated object with many connected joints and links, which forms the 27 degrees of freedom [16] for the hand.Typically the implementation of gesture recognition system required different kinds of devices for capturing and tracking image/ video image [6] such as camera(s), instrumented (data) gloves, and colored marker [6]. Those devices are used for modeling the communication between human and environments rather than traditional interface device such as keyboards, and mice which are inconvenient and unnatural for HCI system. Vision based technique also different according to some system environments such as number of cameras used [6], speed [6], and illumination conditions [6]. The major difficulty in gesture recognition system is how to identify a specific gesture meaning by the machines (computers/ robot) [17].

The purpose of this paper is to present a review of vision based hand gesture recognition techniques for human computer interaction, and to explain various approaches with its advantages and disadvantages. Although recent reviews [6], [7][18][17][19][20][1] in computer vision based have explained the importance of gesture recognition system for human computer interaction (HCI), this work concentrates on vision based techniques method and it's up-to-date. With intending to point out various research developments as well as it represent good starting for interested persons in hand gesture recognition area.

This paper is organized as follows: Section 2 demonstrates hand gesture recognition technology. Vision based hand gesture recognition approaches are given in Section 3. Section 4 explains gesture recognition techniques. Section 5 explains hardware and software implementation tools. Some conclusion and future directions are given in Section 6.

\section{HAND GESTURE TECHNOLOGY}

For any system the first step is to collect the data necessary to accomplish a specific task. For hand posture and gesture recognition system different technologies are used for acquiring input data. Present technologies for recognizing gestures can be divided into vision based, instrumented (data) glove, and colored marker approaches. Figure 1 shows an example of these technologies.

A. Vision Based approaches: In vision based methods the system requires only camera(s) to capture the image required for the natural interaction between human and 
computers and no extra devices are needed. Although these approaches are simple but a lot of gesture challenges are raised such as the complex background, lighting variation, and other skin color objects with the hand object, besides system requirements such as velocity, recognition time, robustness, and computational efficiency [7][17].

B. Instrumented Glove approaches: Instrumented data glove approaches use sensor devices for capturing hand position, and motion. These approaches can easily provide exact coordinates of palm and finger's location and orientation, and hand configurations [17][21][22], however these approaches require the user to be connected with the computer physically [22] which obstacle the ease of interaction between users and computers, besides the price of these devices are quite expensive [22], it is inefficient for working in virtual reality [22].

C. Colored Markers approaches: Marked gloves or colored markers are gloves that worn by the human hand [5] with some colors to direct the process of tracking the hand and locating the palm and fingers [5], which provide the ability to extract geometric features necessary to form hand shape [5]. The color glove shape might consist of small regions with different colors or as applied in [23] where three different colors are used to represent the fingers and palms, where a wool glove was used. The amenity of this technology is its simplicity in use, and cost low price comparing with instrumented data glove [23]. However this technology still limits the naturalness level for human computer interaction to interact with the computer [5].
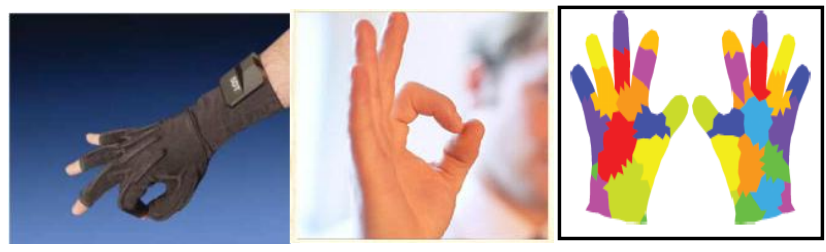

(a) Data-Glove based [21]. (b) Vision based. (c) Colored marker (from web gallery).

Fig 1: Examples of hand gesture recognition input technologies.

\section{VISION BASED HAND GESTURE RECOGNITION APPROACHES}

Vision based technologies use a bare hand to extract data needed for recognition [5], these methods are natural [5][6], easy [6], and the user directly interact with the system [5] . Vision based technology deals with some image characteristics such as texture [6] and color [6] for acquiring data needed for gesture analyze [6]. There are many techniques are applied for detecting hand object after some image preprocessing operations, these methods can be divided into two parts.

\subsection{Appearance Based Approaches}

In appearance based approaches, the visual appearance of the input hand image is modeled using the feature extracted from the image, which will be compared with the features extracted from stored image [15]. Appearance based approaches are simpler and easier than 3D model based approaches due to the easier extraction of features in 2D image [15]. The common method used this approach is to detect skin colored regions in the image; however this method is affect with changing illumination conditions and other background objects with skin like color. At present a lot of study efforts have been grown on approaches that apply invariant features [17], such as AdaBoost learning algorithm [17]. The use of invariant features enables the identification of regions or points on a particular object, rather than modeling the entire objects [17]. With this method the problem of partial occlusion has been overcome [17].

\subsection{D Model Based Approaches}

Model based approaches used 3D model description for modeling and analysis the hand shape [17]. In these approaches search for the kinematic parameters are required by making $2 \mathrm{D}$ projection from $3 \mathrm{D}$ model of the hand to correspond edges images of the hand [7], but a lot of hand features might be lost in 2D projection [7]. 3D Model can be classified into volumetric and skeletal models [16][24]. Volumetric models deal with 3D visual appearance of human hand [16] and usually used in real time applications [5][24]. The main problem with this modeling technique is that it deals with all the parameters of the hand which are huge dimensionality [16]. Skeletal models overcome volumetric hand parameters problem by limiting the set of parameters to model the hand shape from 3D structure [16][5]. Figure 2 shows 3D model approaches.

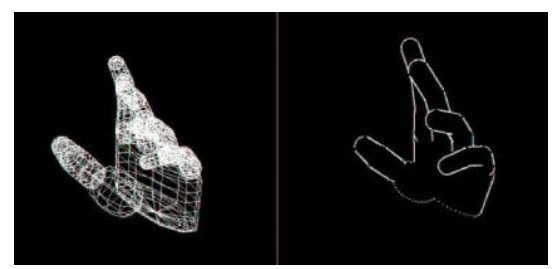

Fig 2: 3D model (left) and the generated contour (right) [17].

In vision based hand gesture recognition system, a video camera used to record hand movements, and the input video is partitioned into frames, for each frame, a set of features are extracted. After some preprocessing operations, the hand object is localized and segmented and the necessary features are extracted and stored in the computer as a trained set. Then each input image pass through the previous steps to extract its features, and classification algorithms are applied by comparing the extracted features from input image with the training set, to interpret the gesture meaning according to a specific application [17]. Figure 3 shows a block diagram of hand gesture recognition system.

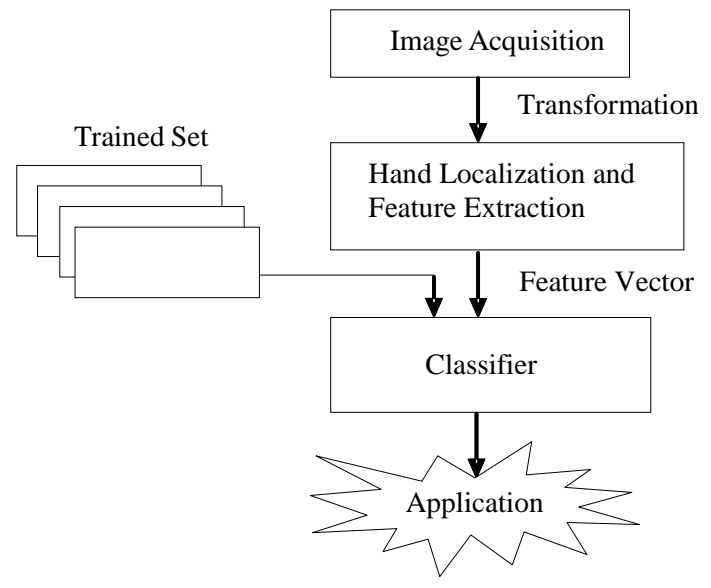

Fig 3: Block diagram of gesture recognition process. 


\section{GESTURE RECOGNITION TECHNIQUES}

The recognition of gesture involves several concepts such as pattern recognition [19], motion detection and analysis [19], and machine learning [19]. Different tools and techniques are utilized in gesture recognition systems, such as computer vision [6], image processing [6], pattern recognition [6], statistical modeling [6].

\section{A. Artificial Neural Networks (ANN)}

The use of neural networks for gesture recognition has been examined by many researchers. Most of the researches use ANN as a classifier in gesture recognition process, while some others use it to extract the shape of the hand, as in [25]. Tin H. [26] presents a system for hand tracking and gesture recognition using NNs to recognize Myanmar Alphabet Language (MAL). Adobe Photoshop filter is applied to find the edges of the input image and histogram of local orientation employed to extract image feature vector which would be the input to the supervised neural networks system. Manar M. [27] used two recurrent neural network architectures to recognize Arabic Sign Language (ArSL). Elman (partially) recurrent neural networks and fully recurrent neural networks have been used separately. A colored glove used for input image data, and for segmentation process, HSI color model is applied. The segmentation divides the image into six color layers, one for the wrist and five for fingertips. 30 features are extracted and grouped to represent a single image, fifteen elements used to represent the angles between the fingertips and between them and the wrist [27], and fifteen elements to represent distances between fingertips; and between fingertips and the wrist [27]. This input feature vector is the input to both neural networks systems. 900 colored images were used as training set, and 300 colored images for system testing. Results had shown that fully recurrent neural network system (with recognition rate 95.11\%) better than the Elman neural network (with $89.67 \%$ recognition rate).

Kouichi M. in [28] presented Japanese sign language recognition using two different neural network systems. Firstly, back propagation algorithm was used for learning postures of Japanese alphabet. For input postures, data glove is used, and normalization operation was applied as a preprocessing step on the input image. The feature extracted from input images was 13 data items, ten for bending, and three for angles in the coordinates. The output of the network was 42 characters. The network consists of three layers, the input layer with 13 nodes, the hidden layer with 100 nodes, and the output layer with 42 nodes which corresponds 42 recognized characters. The recognition rate for learning 42 taught patterns was $71.4 \%$, and for unregistered people $47.8 \%$, while the rate improved when additional patterns added to the system, it became $98.0 \%$ for registered, and $77.0 \%$ for unregistered people.Elman Recurrent Neural Network was the second system applied for recognition gestures. The system could recognize 10 words. The data item have been taken from data glove and the same preprocessing applied for input image. Features extracted are 16 data items, 10 for bending, 3 for angles in the coordinates, and 3 for angles in the coordinates. The network consists of three layers, the input layer with 16 nodes, the hidden layer with 150 nodes, and the output layer with 10 nodes which corresponds 10 recognized words. Some improvement in the positional data and filtering data space are added to the system [28]. Integration of these two neural networks, in a way, that after receiving data from data glove, a determination of the start sampling time and if the data item considered a gesture or a posture is sent to the next network, for checking the sampling data and the system hold a history, which decide the end of sign language. The final recognition rate with the encoding methods was $96 \%$.

Stergiopoulou E. [25] recognized static hand gestures using Self-Growing and Self-Organized Neural Gas (SGONG) network. A camera used for acquiring the input image, and $\mathrm{YCbCr}$ color space is applied to detect hand region, some thresholding technique used to detect skin color. SGONG network use competitive Hebbian learning algorithm for learning process, the learning start with only two neurons and continuous growing till a grid of neurons are constructed and cover the hand object which will capture the shape of the hand. From the resultant hand shape three geometric features was extracted, two angles based on hand slope and the distance from the palm center was determined, where these features used to determine the number of the raised fingers. For recognizing fingertip, Gaussian distribution model used by classifying the fingers into five classes and compute the features for each class. The system recognized 31 predefined gestures with recognition rate $90.45 \%$, in processing time 1.5 second. Shweta K. in [29] introduced gesture recognition system using Neural Networks. Web-cam used for capturing input image at slow rate samples between 15-25 frames per second. Some preprocessing have been made on the input image which convert the input image into sequence of $(x, y)$ coordinates using MATLAB, then passed into neural classifier, in which it will classify the gesture into one of several classed predefined classes which can be identified by the system. Sidney and Geoffiey [30],[31] used neural networks to map hand gestures to speech synthesizer using Glove-Talk system that translated gestures to speech through adaptive interface which is an important class of neural networks applications [31].

\section{B. Histogram Based Feature}

Many researches have been applied based the histogram, where the orientation histogram is used as a feature vector [32]. The first implementation of the orientation histogram in gesture recognition system and real time was done by William F. and Michal R. [32]; they presented a method for recognizing gestures based on pattern recognition using orientation histogram. For digitized input image, black and white input video was used, some transformations were made on the image to compute the histogram of local orientation of each image, then a filter applied to blur the histogram, and plot it in polar coordinates. The system consists of two phases; training phase, and running phase. In the training phase, for different input gestures the training set is stored with their histograms. In running phase an input image is presented to the computer and the feature vector for the new image is formed, Then comparison performed between the feature vector of the input image with the feature vector (oriented histogram) of all images of the training phase, using Euclidean distance metric and the less error between the two compared histograms will be selected. The total process time was $100 \mathrm{msec}$ per frame.

Hanning Z., et al. [33] presented hand gesture recognition system based on local orientation histogram feature distribution model. Skin color based segmentation algorithm were used to find a mask for the hand region, where the input RGB image converted into HSI color space, and then map the HSI image $\mathrm{H}$ to a likelihood ratio image $\mathrm{L}$ the hand region is segmented by thresholding value, 128 elements in the local orientation histogram feature were used. The augmented of the local orientation histogram feature vector implemented by adding the image coordinates of the sub-window. To compact features representation, k-means clustering has been applied 
over the augmented local orientation histogram vectors. In Recognition stage, Euclidean distance used to compute the exact matching score between the input image and stored posture. Then Locality Sensitive Hashing (LSH) used to find the approximate nearest neighbors, and reduce computational cost for image retrieval. Wysoski et al. [34] presented a rotation invariant static-gesture recognition approach using boundary histograms. Skin color detection filter was used, followed by performing erosion, dilation as preprocessing operation, and clustering process to find the groups in the image. For each group the boundary was extracted using an ordinary contour-tracking algorithm. The image Divided into grids, and normalized the boundary in size, which give the system invariance distance between the camera and hand. Homogeneous background was applied, and the boundary is represented as chord's size chain. The image was divided into a number of regions $\mathrm{N}$. And the regions were divided in a radial form [34], according to a specific angle as shown in the Figure. The histogram of boundary chord's size was calculated. So the whole feature vector consists of a sequential chain of histograms. Multilayer perceptron (MLP) Neural Networks and Dynamic Programming (DP) matching were used as classifiers. 26 static postures from American Sign Language, for every posture, 40 pictures were taken, 20 pictures for training and 20 for test. Different number of histograms were used varies from 8 to 36 increasing by two, with different histogram resolutions.

\section{Fuzzy Clustering Algorithm}

Clustering algorithms is a general term comprises all methods that partitioning the given set of sample data into subsets or clusters [35] based on some measures between grouped elements [12]. According to this measure the pattern that share the same characteristics are grouped together to form a cluster [12]. Clustering Algorithms have been widely spread because of their ability of grouping complicated data collections into regularly clusters [35]. In fuzzy clustering, the partitioning of sample data into groups in a fuzzy way are the main difference between fuzzy clustering and other clustering algorithm [12], where the single data pattern might belong to different data groups [12].

Xingyan L. In [12] presented fuzzy c-means clustering algorithm to recognize hand gestures in a mobile remote. A camera was used for acquire input raw images, the input RGB images are converted into HSV color model, and the hand extracted after some preprocessing operations to remove noise and unwanted objects, and thresholding used to segment the hand shape. 13 elements were used as feature vector, first one for aspect ratio of the hand's bounding box, and the rest 12 parameters represent grid cell of the image, and each cell represents the mean gray level in the 3 by 4 blocks partition of the image, where the mean value of each cell represents the average brightness of those pixels in the image, Then FCM algorithm used for classification gestures. Various environments are used in the system such as complex background and invariant lighting conditions. 6 hand gestures used with 20 samples for each gesture in the vocabulary to create the training set, with recognition accuracy $85.83 \%$.

\section{Hidden Markov Model (HMM)}

Many researches were applied in the field of gesture recognition using HMM. HMM is a stochastic process [6], with a finite number of states of Markov chain, and a number of random functions so that each state has a random function [6]. HMM system topology is represented by one state for the initial state, a set of output symbols [6][22], and a set of transitions state [22][8]. HMM contained a lot of mathematical structures and has proved its efficiency for modeling spatio-temporal information data [6]. Sign language recognition, are one of the most applications of HMM [8], and speech recognition [10].

In [9] Keskiin C., et. al. presented HCI interface based on real time hand tracking and 3D gesture recognition using hidden Markov models (HMM). Two colored cameras for 3D construction are used. To overcome the problem of using skin color for hand detection because of hand overlapping with other body parts, markers are used to reduce the complexity in hand detection process [9]. Markers used to segment the hand from complex backgrounds under invariant lighting conditions. The markers are distinguished using marker detection utility, and connected components algorithm was applied to find marker regions using double thresholding. For fingertip detection, simple descriptors were used, where the bounding box and four outmost points of the hand that defining the box is determined [9]. The bounding box in some cases needs to be elongate to determine the mode of the hand, and the points used to predict the fingertip location in different modes of the hand. Kalman filter was used for filtering trajectory of the hand motion. For 3D reconstruction of finger coordinates, calibration utility was implemented for specific calibration object [9]. Least square approach used to generate fingertip coordinates, and kalman filter applied for smoothing the trajectory of $3 \mathrm{D}$ reconstructed coordinates. To eliminate coordinate system dependency, the 3D coordinates are converted into sequences of quantized velocity vectors. HMM interprets these sequences [9], which are directional characterizing of the motion [9]. The system designed for game and painting programs application. Hand tracking is utilized to imitate the movements of the mouse for drawing, and the gesture recognition system used for selecting commands. Eight gestures have been used for system training, and 160 for testing, with $98.75 \%$ recognition performance.

\section{IMPLEMENTATION TOOLS}

Many implementation hardware and software tools have been utilized for recognizing gestures depending on the application fields used.

\subsection{Hardware Implementation Tools}

Input devices used for gesture recognition systems are various and different according to the system and application used for recognition process. Single camera can be used for postures recognition since this environment might be inconvenient for other types of image-based recognition [24]. Stereo camera which consists of two lenses with an isolated sensor for each lens [24], which imitates human visual system therefore, the $3 \mathrm{D}$ effect of views is created [24]. Stereo camera can be used to make 3D pictures for movies [24], or for range imaging [24].

Tracking device such as instrumented data gloves measure finger movements through various types of sensors technology [21], [22]. It can provide accurate information about the position and orientation of the hands using magnetic or inertial tracking devices [24]. For more details about various types of glove-based input device refer to [21], [22].

Controller-based gestures, in this type of input gestures, controllers represent a complement of the human, so that when the body moves to perform some gestures [24], these motions are captured using some software [24]. Mouse gestures are an example of such controllers [24]. Other systems based on accelerometers to measure hand movements [36] [37]. 
Table 1.Comparisons between various gesture recognition systems.

\begin{tabular}{|c|c|c|c|c|c|c|c|}
\hline Method & $\begin{array}{l}\text { Type Of } \\
\text { Input } \\
\text { Device }\end{array}$ & $\begin{array}{c}\text { Segmentation } \\
\text { Type }\end{array}$ & $\begin{array}{l}\text { Features } \\
\text { (Geometric } \\
\text { Or Non) }\end{array}$ & $\begin{array}{l}\text { Feature Vector } \\
\text { Representation }\end{array}$ & \multicolumn{2}{|c|}{$\begin{array}{c}\text { Classification } \\
\text { Algorithm }\end{array}$} & $\begin{array}{c}\text { Recognition } \\
\text { Rate }\end{array}$ \\
\hline Tin H. [26] & $\begin{array}{l}\text { Digital } \\
\text { camera }\end{array}$ & threshold & Non geometric & $\begin{array}{l}\text { Orientation } \\
\text { histogram }\end{array}$ & \multicolumn{2}{|c|}{$\begin{array}{c}\text { supervised neural } \\
\text { network }\end{array}$} & $90 \%$ \\
\hline \multirow{2}{*}{ Manar M. [27] } & \multirow{2}{*}{$\begin{array}{l}\text { Colored } \\
\text { glove, and } \\
\text { Digital } \\
\text { camera }\end{array}$} & \multirow{2}{*}{ HSI color model } & \multirow{2}{*}{ N/A } & \multirow{2}{*}{$\begin{array}{l}\text { Available } \\
\text { Features from } \\
\text { resource }\end{array}$} & \multirow{2}{*}{$\begin{array}{l}\text { Two } \\
\text { neural } \\
\text { networ } \\
\mathrm{k} \\
\text { system }\end{array}$} & $\begin{array}{c}\text { Elman } \\
\text { recurrent } \\
\text { network }\end{array}$ & $89.66 \%$ \\
\hline & & & & & & $\begin{array}{c}\text { Fully } \\
\text { recurrent } \\
\text { network }\end{array}$ & $95.11 \%$ \\
\hline \multirow[b]{2}{*}{$\begin{array}{l}\text { Kouichi M. } \\
\text { [28] }\end{array}$} & \multirow[b]{2}{*}{$\begin{array}{l}\text { Data } \\
\text { glove }\end{array}$} & \multirow[b]{2}{*}{ threshold } & \multirow[b]{2}{*}{ Non geometric } & $\begin{array}{c}13 \text { data item (10 } \\
\text { for bending, } 3 \\
\text { for coordinate } \\
\text { angles) }\end{array}$ & \multirow{2}{*}{$\begin{array}{l}\text { Two } \\
\text { neural } \\
\text { networ } \\
\mathrm{k} \\
\text { system }\end{array}$} & $\begin{array}{l}\text { back } \\
\text { propagation } \\
\text { network }\end{array}$ & $71.4 \%$ \\
\hline & & & & $\begin{array}{l}16 \text { data item (10 } \\
\text { for bending, } 3 \\
\text { for coordinate } \\
\text { angles, } 3 \text { for } \\
\text { positional data) }\end{array}$ & & $\begin{array}{l}\text { Elman } \\
\text { recurrent } \\
\text { network }\end{array}$ & $96 \%$ \\
\hline $\begin{array}{l}\text { Stergiopoulou } \\
\text { E. [25] }\end{array}$ & $\begin{array}{l}\text { Digital } \\
\text { camera }\end{array}$ & YCbCr color space & geometric & $\begin{array}{c}\text { Two angles of } \\
\text { the hand shape, } \\
\text { compute palm } \\
\text { distance }\end{array}$ & \multicolumn{2}{|c|}{ Gaussian distribution } & $90.45 \%$ \\
\hline Shweta in [29] & $\begin{array}{l}\text { Web- } \\
\text { cam }\end{array}$ & & Non geometric & & \multicolumn{2}{|c|}{$\begin{array}{c}\text { supervised neural } \\
\text { network }\end{array}$} & N/A \\
\hline $\begin{array}{l}\text { William F. and } \\
\text { Michal R. [32] }\end{array}$ & $\begin{array}{l}\text { Digital } \\
\text { camera }\end{array}$ & & Non geometric & $\begin{array}{c}\text { Orientation } \\
\text { histogram }\end{array}$ & \multicolumn{2}{|c|}{$\begin{array}{l}\text { Euclidean distance } \\
\text { metric }\end{array}$} & N/A \\
\hline $\begin{array}{l}\text { Hanning Z., et } \\
\text { al. [33] }\end{array}$ & $\begin{array}{l}\text { Digital } \\
\text { camera }\end{array}$ & threshold & Non geometric & $\begin{array}{c}\text { augmented of the } \\
\text { local orientation } \\
\text { histogram }\end{array}$ & \multicolumn{2}{|c|}{$\begin{array}{l}\text { Euclidean distance } \\
\text { metric }\end{array}$} & $92.3 \%$. \\
\hline $\begin{array}{c}\text { Wysoski, et al. } \\
{[34]}\end{array}$ & $\begin{array}{l}\text { Digital } \\
\text { camera }\end{array}$ & $\begin{array}{c}\text { skin color } \\
\text { detection filter } \\
\end{array}$ & Geometric & $\begin{array}{l}\text { the histogram of } \\
\text { radial boundary }\end{array}$ & \multicolumn{2}{|c|}{ MLP+ DP matching } & $98.7 \%$ \\
\hline $\begin{array}{c}\text { Xingyan L. } \\
\text { [12] }\end{array}$ & $\begin{array}{l}\text { Digital } \\
\text { camera }\end{array}$ & threshold & Non geometric & $\begin{array}{c}\text { One dimensional } \\
\text { array of } 13 \\
\text { element }\end{array}$ & \multicolumn{2}{|c|}{$\begin{array}{l}\text { Fuzzy C-Means } \\
\text { algorithm }\end{array}$} & $85.83 \%$ \\
\hline $\begin{array}{l}\text { Keskiin C., et } \\
\text { al. [39] }\end{array}$ & $\begin{array}{l}\text { Two } \\
\text { colored } \\
\text { cameras } \\
\text { and } \\
\text { marker }\end{array}$ & $\begin{array}{c}\text { connected } \\
\text { components } \\
\text { algorithm with } \\
\text { double } \\
\text { thresholding } \\
\end{array}$ & Geometric & $\begin{array}{l}\text { sequences of } \\
\text { quantized } \\
\text { velocity vectors }\end{array}$ & \multicolumn{2}{|c|}{ HMM } & $98.75 \%$ \\
\hline
\end{tabular}

\subsection{Software Implementation Tools}

Software tools are the programming language and windows system used for implementing the gesture recognition system. Some researches applied programming languages like $\mathrm{C}, \mathrm{C}++$, and Java language. To simplify the work especially when image processing operations are needed, MATLAB ${ }^{\circledR}$ with image processing toolbox is used. Tin H. [26] used $M A T L A B \otimes$ for hand tracking and gesture recognition. Manar M. [27] use MATLAB6 and C language, MATLAB6 used for image segmentation while $\mathrm{C}$ language for Hand Gesture Recognition system. Kouichi [28] use SUN/4 workstation for Japanese Character and word recognition. Also Stergiopoulou [25] used Delphi language with $3 \mathrm{GHs}$ CPU to implement hand gesture recognition system using SGONG network. Shweta [29] used MATLAB® for hand recognition. Freeman and Michal Roth [32] used HP 735 workstation was used for implementing the system. Hanning Zhou et. al. [33] used C++ implementation costs only $1 / 5$ second for the whole preprocessing, feature extraction and recognition, when running on a $1.3 \mathrm{G}$ Intel Pentium laptop processor with $512 \mathrm{MB}$ memory.

\section{CONCLUSION \& FUTUREWROK}

Building an efficient human-machine interaction is an important goal of gesture recognition system. Many applications of gesture recognition system ranging from virtual reality to sign language recognition and robot control. In this paper a survey on tools and techniques of gesture recognition system have been provided with emphasis on hand gesture expressions. The major tools surveyed include HMMs, ANN, and fuzzy clustering have been reviewed and analyzed. Most researchers are using colored images for achieving better results.

Comparison between various gesture recognition systems have been presented with explaining the important parameters needed for any recognition system which include: the segmentation process, features extraction, and the classification algorithm. 


\section{REFERENCES}

[1] John Daugman, 1997. Face and Gesture Recognition: Overview, IEEE transaction on pattern analysis and machine intelligence, vol. 19(7).

[2]Sanjay Meena, 2011. A Study on Hand Gesture Recognition Technique, Master thesis, Department of Electronics and Communication Engineering, National Institute of Technology, India.

[3] Myers, B.A., 1988. A Taxonomy of User Interfaces for Window Managers. IEEE Transaction in Computer Graphics and Applications, 8(5), pp. 65-84.Doi; $10.1109 / 38.7762$

[4] Myers B. A., 1998. A Brief History of Human Computer Interaction Technology, ACM interactions. pp. 44-54, Vol. 5(2). Doi: 10.1145/274430.274436

[5] Mokhtar M. Hasan, and Pramod K. Mishra, 2012. Hand Gesture Modeling and Recognition using Geometric Features: A Review, Canadian Journal on Image Processing and Computer Vision Vol. 3, No.1.

[6] S. Mitra, and T. Acharya, 2007. Gesture Recognition: A Survey. IEEE Transactions on systems, Man and Cybernetics, Part C: Applications and reviews, vol. 37 (3), pp. 311-324, doi: 10.1109/TSMCC.2007.893280.

[7] G. R. S. Murthy \& R. S. Jadon, 2009. A Review if Vision Based Hand Gestures Recognition, International Journal of Information Technology and Knowledge Management, vol. 2(2), pp. 405-410.

[8] Thad Starner and Alex Pentland, 1996. Real-Time American Sign Language Recognition from Video Using Hidden Markov Models, AAAI Technical Report FS-9605, The Media Laboratory Massachusetts Institute of Technology.

[9] C. Keskin, A. Erkan, L. Akarun, 2003. Real Time Hand Tracking and 3D Gesture Recognition for Interactive Interfaces using HMM, In Proceedings of International Conference on Artificial Neural Networks.

[10] Lawrence R. Rabiner, 1989. A Tutorial on Hidden Markov Models and Selected Applications in Speech Recognition, Proceedings of the IEEE, vol. 77 (2), pp. 257 - 286.

[11] Pengyu H., Matthew T., Thomas S. Huang. 2000. Constructing Finite State Machines for Fast Gesture Recognition, IEEE Proceedings, 15th International Conference on Pattern Recognition (ICPR 2000), vol. 3 ,pp. 3691-694, 2000, doi:10.1109/ICPR.2000.903639.

[12] Xingyan Li, 2003. Gesture Recognition based on Fuzzy C-Means Clustering Algorithm, Department of Computer Science. The University of Tennessee. Knoxville.

[13] David E. Goldberg, 1989. Genetic Algorithms in Search, Optimization, and Machine Learning, Edition 1.

[14] Ben Krose, and Patrick van der Smagtan, 1996.An introduction to Neural Networks, the University of Amsterdam, eighth edition.

[15] sara Bilal, RiniAkmeliawati, Momoh J. El Salami, Amir A. Shafie, 2011. Vision-Based Hand Posture Detection and Recognition for sign Language - A study, IEEE 4th international conference on Mechatronics (ICOM 2011), pp. 1-6.
[16] Vladimir I. Pavlovic, Rajeev Sharma, and Thomas S. Huang, 1997. Visual Interpretation of Hand Gestures for Human-Computer Interaction: A Review, IEEE Transactions On Pattern Analysis And Machine Intelligence, vol. 19(7), pp. 677- 695.

[17] PragatiGarg, Naveen Aggarwal and SanjeevSofat, 2009. Vision Based Hand Gesture Recognition, World Academy of Science, Engineering and Technology 49, pp. 972-977.

[18] Thomas B. Moeslund and Erik Granum, 2001. A Survey of Computer Vision-Based Human Motion Capture, Elsevier, Computer Vision and Image Understanding 81, Ideal, pp. 231-268.

[19] Ying Wu, Thomas S. Huang, 1999. Vision-Based Gesture Recognition: A Review, Lecture Notes in Computer Science, Gesture Workshop, proceedings of the international Gesture Workshop on Gesture-Based communication in Human-Computer interaction, vol.(1739), pp. 103-115.

[20] Ali Erol, George Bebis, MirceaNicolescu, Richard D. Boyle, XanderTwombly, 2007. Vision-based hand pose estimation: A review, Elsevier Computer Vision and Image Understanding 108, pp. 52-73.

[21] Laura Dipietro, Angelo M. Sabatini, and Paolo Dario, 2008. Survey of Glove-Based Systems and their applications, IEEE Transactions on systems, Man and Cybernetics, Part C: Applications and reviews, vol. 38(4), pp. 461-482, doi: 10.1109/TSMCC.2008.923862

[22] Joseph J. LaViola Jr. 1999. A Survey of Hand Posture and Gesture Recognition Techniques and Technology, Master Thesis, NSF Science and Technology Center for Computer Graphics and Scientific Visualization, USA.

[23] Luigi Lamberti\& Francesco Camastra, 2011. Real-Time Hand Gesture Recognition Using a Color Glove, Springer 16th international conference on Image analysis and processing: Part I (ICIAP'11), pp. 365-373.

[24] Wikipedia websit.

[25] E. Stergiopoulou, N. Papamarkos, 2009. Hand gesture recognition using a neural network shape fitting technique, Elsevier Engineering Applications of Artificial Intelligence 22, pp. 1141-1158.

[26] Tin Hninn H. Maung, 2009. Real-Time Hand Tracking and Gesture Recognition System Using Neural Networks, World Academy of Science, Engineering and Technology 50, pp. 466- 470 .

[27] ManarMaraqa, Raed Abu-Zaiter, 2008. Recognition of Arabic Sign Language (ArSL) Using Recurrent Neural Networks, IEEE First International Conference on the Applications of Digital Information and Web Technologies, ICADIWT, Aug. 2008, pp. 478-48, doi: 10.1109/ICADIWT.2008.4664396

[28] Kouichi Murakami and Hitomi Taguchi, 1999. Gesture Recognition using Recurrent Neural Networks, ACM, pp. 237-242.

[29] Shweta K. Yewale, 2011. Artificail Neural Network Approach For Hand Gesture Recognition, International Journal of engineering Science and Technology (IJEST), vol. 3(4). 
[30] S. Sidney Fels, Geoffiey E. Hinton, 1993. Glove-Talk: A Neural Network Interface Between a Data-Glove and a Speech Synthesizer, IEEE transaction on Neural Networks, vol. 4(1), pp. 2-8, doi: 10.1109/72.182690

[31] S. Sidney Fels, Geoffiey E. Hinton, 1998. GloveTalkII-A Neural-Network Interface which Maps Gestures to Parallel FormantSpeech Synthesizer Controls, IEEE transactions on neural networks, vol. 9(1), pp. 205-212, doi: 10.1109/72.655042

[32] William T. Freeman and Michal Roth, 1995. Orientation Histograms for Hand Gesture Recognition, IEEE International Workshop on Automatic Face and Gesture Recognition, Zurich.

[33] Hanning Zhou, Dennis J. Lin and Thomas S. Huang, 2004. Static Hand Gesture Recognition based on Local Orientation Histogram Feature Distribution Model, Proceedings of the IEEE Computer Society Conference on Computer Vision and Pattern Recognition Workshops (CVPRW'04).
[34] Simei G. Wysoski, Marcus V. Lamar, Susumu Kuroyanagi , Akira Iwata, 2002. A rotation invariant approach on static-gesture recognition using boundary histograms and neural networks, IEEE Proceedings of the 9th International Conference on Neural Information Processing, Singapura, November.

[35] James C. Bezdek, Robert Ehrlich, William Full, 1984. FCM: The Fuzzy C-Means Clustering Algorithm, Computers \& Geosciences vol. 10(2-3), pp. 191-203.

[36] Damien Zufferey, 2008. Device based gesture recognition, ACM Second International Conference on Tangible and. Embedded Interaction (TEI'08). pp.

[37] Marco Klingmann, 2009. Accelerometer-Based Gesture Recognition with the iPhone, Master Thesis in Cognitive Computing, Goldsmiths University of London. 